\title{
Knockdown of Long Non-Coding RNA GAS5 Increases miR-23a by Targeting ATG3 Involved in Autophagy and Cell Viability
}

\author{
Lexing Li Chunxiao Huang Yulong He Zhan Sang Guoquan Liu \\ Hanchuan Dai \\ College of Veterinary Medicine, Huazhong Agricultural University, Wuhan, China
}

\section{Key Words}

Lncrna GAS5 • $\mathrm{miR}-23 \mathrm{a} \cdot \mathrm{ATG} 3 \cdot$ Autophagy $•$ Cell viability

\begin{abstract}
Background/Aims: Autophagy is a process of evolutionarily conservative degradation, which could maintain cellular homeostasis and cope with various types of stress. LncRNAs are considered as competing endogenous RNAs (ceRNAs) contributing to autophagy. GAS5 has been suggested as a new potential factor to mediate autophagy pathway and the underlying mechanism remains to be further confirmed. This study was taken to identify the effect of GAS5/miR-23a/ATG3 axis on autophagy and cell viability. Methods: The western blotting assay was used to detecte the protein levels of LC3, mTOR, Beclin-1, ATG3, ATG5-ATG12 complex and p62. The mRNA level of Pre-miR-23a, Pri-miR-23a, miR-23a, GAS5, LC3, mTOR and ATG3 were quantified by real-time RT-PCR. Dual-luciferase reporter assays were performed to confirm the direct binding of miR-23a and ATG3 or GAS5. Cell viability was evaluated by CCK-8 and flow cytometry. Results: We showed that miR-23a could directly suppress ATG3 expression in 293T cells, which suggested that ATG3 was identified as a target of miR-23a. MiR-23a mimics could restrain LC3 II, Beclin1 levles and ATG5-ATG12 complex formation. Meanwhile, miR-23a also increased the expression of mTOR and p62. Notably, there was a putative miR-23a-binding site in GAS5. MiR-23a overexpression might suppress the GAS5 expression, but the repressive effect was abolished by mutation of binding sites. Importantly, overexpression of GAS5 could inhibit the mature miR-23a and has no effect on miR-23a precursors. Knockdown of GAS5 suppressed the expression of LC3 II, ATG3 and ATG5-ATG12 complex formation, whereas p62 and mTOR levels were promoted. The further results showed that miR-23a overexpression and GAS5 inhibition both significantly suppressed cell viability and promoted the apoptosis rate following LPS stimulation, and knockdown of miR-23a exhibited the opposite effects. Conclusions: Our study revealed that down-regulation GAS5 attenuated cell viability and inhibited autophagy through ATG3-dependent autophagy by regulating miR-23a expression. The results suggested that GAS5/miR-23a/ATG3 axis might be a novel regulatory network contributing to a better understanding of regulation on autophagy program and cell viability.




\section{Cellular Physiology Cell Physiol Biochem 2018;48:1723-1734 \\ \begin{tabular}{l|l} 
and Biochemistry Published online: August 2, 2018 & $\begin{array}{l}\text { (c) } 2018 \text { The Author(s). Published by S. Karger AG, Basel } \\
\text { www.karger.com/cpb }\end{array}$
\end{tabular} \\ Li et al.: IncRNA GAS5/miR-23a Axis Was Involved in ATG3-Dependent Autophagy and}

Cell Viability

\section{Introduction}

Autophagy is an evolutionarily conserved process that breaks down damaged organelles and macromolecules through lysosomal-dependent degradation, and it is involved in a variety of vital movements including cell homeostasis, cancer, heart failure, aging and neurodegenerative diseases [1-3]. More than 30 autophagy related proteins (ATG) are essential drivers of autophagy pathways [4]. During autophagy, microtubuleassociated protein 1 light chain 3-I (LC3-I) is shifted to E2-like enzyme ATG3 connected with phosphatidyl ethanolamine (PE), and then lipidated to LC3- II with the help of ATG5-ATG12ATG16 complex, which contributes to promote autophagosome formation. The conversion from a cytosolic form of LC3-I to LC3-II, has been shown to be an autophagosomal marker in mammals $[5,6]$. Beclin1 is a phylogenetically conserved protein that is an essential regulator for autophagy [7]. It functions as a scaffold protein to constitute the class III PI3K (phosphoinositide 3-kinase) complex, promoting autophagy by recruiting ATG9, ATG14L and UVRAG into autophagosome membrane [8,9]. Furthermore, LC3 can trap p62 and selectively transport it into the autophagosome. Accumulation of p62 has been used as a marker for inhibition of autophagy or defect in autophagic degradation [10]. As suggested, LC3 II and Beclin1 were autophagy markers to monitor the autophagy flux, and p62 was an autophagic substrates to verify the lysosome degradation [11]. Although, great efforts have been made to understand the basic process and molecular mechanism, the detailed mechanism of autophagy remains to be elucidated.

As researches have progressed, approximately $90 \%$ of human genome is extensively transcribed, but only $2 \%$ of them serve as protein-coding genes. The majority of the remaining transcripts with no protein-coding capacity are transcribed into non-coding RNAs (ncRNAs), especially microRNAs (miRNAs) and long non-coding RNAs (lncRNAs) [12]. MiRNAs have been indicated to be involved in regulating various physiology processes, such as cellular proliferation, differentiation, as well as apoptosis predominantly through base pairing with the 3'-UTR of target mRNA $[13,14]$. It is well documented that miRNAs function as autophagy regulator via specifically regulating autophagy related genes $[2,15$ 17]. MiR-23a, belonging to miR-23a 24-2 27a gene cluster, functions in cell differentiation, tumor growth and metastasis [18-20]. Recently, miR-23a was found to be an inducer of autophagy. Down-regulated miR-23a contributed to the metastasis of cutaneous melanoma by promoting autophagy through directly targeting ATG12 [21]. Forced expression of miR23a-5p inhibited the activation of autophagy through TLR2/MyD88/NF- $\kappa B$ pathway by targeting TLR2 [22]. Besides, antagomir-mediated inactivation of miR-23a resulted in the stimulation of PUVA- and UVB-depressed autophagy flux and protected human fibroblasts from premature senescence by targeting AMBRA1 [23]. Although accumulating studies have demonstrated that miR-23a participates in the regulation of autophagy, the role of miR-23a in the autophagy response has yet been fully elucidated.

Long non-coding RNAs (lncRNAs) are a class of RNAs with molecular weight of more than 200 bases in length and initially reported to participate in various cellular process, including mRNA stability, post-transcriptional regulation, chromosome modification [24]. Also, emerging studies have implicated that IncRNAs are considered as competing endogenous RNAs (ceRNAs) contributing to autophagy. Wang et al. found that lncRNA APF regulates autophagy and myocardial infarction though sponging miR-188-3p [25], and lncRNA FLJ11812 activated autophagy through sponging miR-4459 in vascular endothelial cells [26]. In addition, IncRNA HOTAIR was reported to activate autophagy for promoting hepatocellular carcinoma cell proliferation by upregulating ATG3 and ATG7 expression [27]. BRAF-activated IncRNA contributed to cell proliferation and apoptosis by activating autophagy in papillary thyroid carcinoma [28]. Accumulating evidences demonstrated that IncRNA growth arrest-specific 5 (GAS5) acted as a powerful regulator of various biological activities, including tumor metastasis [29], vascular remodeling [30], autoimmune and 


\section{Cellular Physiology Cell Physiol Biochem 2018;48:1723-1734 and Biochemistry Published online: August 2, $2018 \quad \begin{aligned} & \text { DOI: 10.1159/000492300 } 2018 \text { The Author(s). Published by S. Karger AG, Basel } \\ & \text { www.karger.com/cpb }\end{aligned}$ \\ Li et al.: IncRNA GAS5/miR-23a Axis Was Involved in ATG3-Dependent Autophagy and Cell Viability}

inflammatory diseases [31]. Recently, GAS5 has been reported to involve in autophagy in NSCLC cells [32], and negatively correlated with mTOR [33,34], which is a crucial regulator of autophagy [35]. Although GAS5 has been suggested to mediate autophagy program, the underlying mechanism still remains to be further confirmed.

In our study, we investigated that miR-23a participated in autophagy by suppressing ATG3 transcription and translation. Knockdown of GAS5 inhibited autophagy related gene expression and increased mTOR and p62 levels. We further suggested that GAS5 might directly bind to miR-23a and a mutual negative correlation between GAS5 and miR-23a was received. Also, the function in autophagy modulating by GAS5-miR-23a interaction was investigated. Additionally, our data indicated that GAS5 and miR-23a could influence the proliferation and apoptosis in 293T cells following LPS stimulation. Based on these findings, we hypothesized that GAS5/miR-23a/ATG3 axis might exhibit a crucial role in autophagy regulation and cell viability.

\section{Materials and Methods}

Cell culture and Transfection

$2 \times 10^{5} 293$ T cells were plated into six-well plates and cultured in DMEM (Cat.no: SH3002201, Hyclone, USA) medium supplemented with 10\% fetal bovine serum (Cat.no: 1027-106, Gibco, USA), 100 $\mathrm{U} / \mathrm{mL}$ penicillin, and $100 \mathrm{mg} / \mathrm{mL}$ streptomycin (Invitrogen) in humidified air at $37{ }^{\circ} \mathrm{C}$ with $5 \% \mathrm{CO}_{2}$. Cells at a confluence of $70-80 \%$ were transfected mimics and inhibitor at a final concentration of $50 \mathrm{nM}$ using the Lipofectamine 2000 (Cat.no: 1756108, Invitrogen, USA) according to the manufacturer's instructions. MiR-23a mimics/mimics NC and miR-23a inhibitor/inhibitor NC were commercially synthesized by Genepharma (Shanghai, China). Specific siRNA against GAS5 (5'-GCAAGCCUAACUCAAGCCAdTdT-3', 3'-dTdT CGUUCGGAUUGAGUUCGGU-5') was synthesized by RiboBio (Cat.no: siG1371095114, Guangzhou, China).

\section{Plasmid construction}

The 3'-untranslated region (3'-UTR) of ATG3 fragment containing putative binding

sites (ATGTGA) for miR-23a was amplified by PCR (sense: 5'-CCTCGAGTGAAGAGCACATAGAATCTATC-3'; antisense: 5'-TTGCGGCCGCGCAATCTGAAGTGACACTC-3') from human genomic DNA and cloned into psiCHECK $^{\mathrm{TM}}$-2 dual luciferase reporter plasmid (Promega) to construct psiCHECK ${ }^{\mathrm{TM}}$-2-WT-ATG3 (WT-ATG3). The predicted target site was mutated to CGAGTG and the recombinant plasmid psiCHECK ${ }^{\mathrm{TM}}$-2-MT-ATG3 (MT-ATG3) was constructed by mutagenesis PCR. The complementary DNA encoding GAS5 (exon 4-intron 4-exon 5) was synthesized by Tsingke (Beijing, China) and subcloned into the pcDNA3.1 vector. LncRNAGAS5 (exon 4-intron 4-exon 5) or its point mutations in specific miR-23a binding sites was also cloned into psiCHECK $^{\mathrm{TM}}$ - 2 vector. The luciferase plasmid of psiCHECK $^{\mathrm{TM}}$-2-GAS5-WT (GAS5-WT) or psiCHECK ${ }^{\mathrm{TM}}$-2-GAS5MT (GAS5-MT) was respectively produced.

Quantitative real-time PCR ( $q P C R$ )

Total RNAs from cells were extracted after transfected $24 \mathrm{~h}$ using Trizol reagent (Invitrigen) and $1 \mu \mathrm{g}$ RNA was reverse transcribed with reverse transcriptase (Toyobo) using the stem-loop RT primer (U6: 5' ${ }^{\prime}$ GTCGTATCCAGTGCAGGGTCCGAGGTGCACTGGATACGACAAAATATGG-3', miR-23a: 5'-GTCGTATCCAGTGCAGGGTCCGAGGTATTCGCACTGGATACGACGGAAATC-3') and oligodT (Qingke, China). The RT products were quantified using SYBR Greenreal-time PCR (Cat.no: QPK-201, Toyobo) on the LightCycler® 96 real-time PCR detection system (Roche, Switzerland). Comparative quantification was calculated utilizing the $2^{-\Delta \Delta \mathrm{Ct}}$ method and normalized to U6 or GAPDH levels. All samples were performed in triplicate. Specific primers (Qingke, China) used are as follows (Table 1).

Western blotting assays

The cells were lysed in RIPA buffer (Cat.no: PP1202, Aidelai, China) for 50 min on ice. The total protein concentration of the cell lysate was determined by the bicinchoninic acid method (Cat.no: PP0101, Aidelai, China). The proteins were separated by $8 \%$ or $12 \%$ polyacrylamide gel electrophoresis containing $0.1 \%$ SDS and transferred to PVDF membranes. The membranes were incubated for $2 \mathrm{~h}$ at room temperature in 


\section{Cellular Physiology Cell Physiol Biochem 2018;48:1723-1734

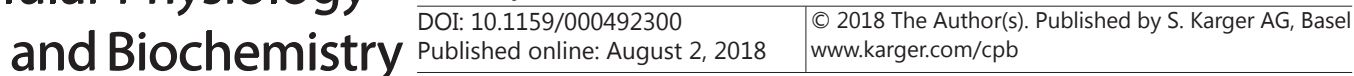 \\ Li et al.: IncRNA GAS5/miR-23a Axis Was Involved in ATG3-Dependent Autophagy and Cell Viability}

blocking buffer (20 mM Tris-HCl, $137 \mathrm{mM} \mathrm{NaCl}$, pH 8.0 , containing $0.1 \%$ Tween and $5 \%$ non-fat dry milk) and probed with antibodies against mTOR (1:500, SAB4501038, Sigma, USA), ATG3 (1:1000, 3415, CST, USA), ATG12 (1:1000, 4180P, CST, USA), p62 (1:1000, 5114S, CST, USA), LC3 (1:1000, 12741, CST, USA), Beclin1 (1:1000, 3495, CST, USA) and GAPDH (1:3000, GB12002, Servicebio, China), $\beta$-tubulin (1:3000, GB130172 , Servicebio, China) at $4{ }^{\circ} \mathrm{C}$ overnight. After being washed 3 times with TBST, the membrane was incubated with HRP-conjugated anti-IgG (1:3000, GB23303, Servicebio, China) at room temperature for $2 \mathrm{~h}$. GAPDH or $\beta$-tubulin was used as an internal control. The reacted proteins were visualized using an electrochemiluminescence (ECL) system (Biotanon, China) and protein ratios were calculated following Image J densitometric analysis.

\section{Luciferase activity assays}

$3 \times 10^{4} 293 \mathrm{~T}$ cells were plated in 24 -well plates and cultured for overnight at $37^{\circ} \mathrm{C}$. Subsequently, cells at a confluence of 70-80\% were co-transfected either WT-ATG3 or MT-ATG3 (50 ng) and miR-23a mimics or negative control mimics (50 $\mathrm{nM}$ ). At the same time, GAS5-WT and GAS5-MT (50 ng) were respectively co-transfected either specific miR-23a mimics or the negative control mimics ( $50 \mathrm{nM}$ ) using Lipofectamine 2000. Firefly and renilla luciferase activities were measured consecutively using the dual-luciferase reporter assay system kit (Cat.no: E1910, Promega) at $48 \mathrm{~h}$ after transfection. Luciferase activity was used as normalization.

\section{Cell proliferation assays}

Cell proliferation assays were carried out using Cell Counting Kit-8 (CCK-8) kit (Cat.no: BS350B, Biosharp, China). 293T ( $5 \times 10^{3} /$ well) cells were plated in 96-well and cultured overnight. At a confluence of 70-80\%, cells were then transfected with miR-23a mimics/mimics NC, miR-23a inhibitor/inhibitor NC or siRNA-GAS5/si-NC (50 nM). After transfection for $24 \mathrm{~h}$, cells were stimulated with LPS (1 $\mu \mathrm{g} / \mathrm{mL})$ for $24 \mathrm{~h}$, and then incubated at $37^{\circ} \mathrm{C}$ for $2 \mathrm{~h}$ by the addition of CCK-8 reagent according to the manufacturer's protocol. The relative viability was determined by calculating the $\mathrm{OD}_{450}$ of cells relative to that of mocktransduced cells.

\section{Apoptosis assays}

Cell apoptosis was determined using Annexin V-FITC/PI kit (Cat.no: KGA108, Keygen, China). Briefly, transfected 293T cells were seeded into 6-well plates at a density of $2 \times 10^{5} /$ well, and transiently transfected with miR-23a mimics/mimics NC, miR-23a inhibitor/inhibitor NC or siRNA-GAS5/si-NC (50 nM). The cells were treated with $1 \mu \mathrm{g} / \mathrm{mL}$ LPS (Sigma) for $4 \mathrm{~h}$ after transfection for $20 \mathrm{~h}$. The collected cells were incubated at room temperature for $15 \mathrm{~min}$ in the dark with FITC-Annexin $\mathrm{V}$ and propidium iodide (PI) solution according to the manufacturer's instructions. Cells were analyzed using flow cytometry (BectonDickinson, USA).

\section{Statistical analysis}

Data were expressed as the mean \pm s.e.m. of at least three independent experiments for each cellular experimental group. We evaluated the data by two-tailed student's t-test with Graphpad prism version 5.0 (Graphpad software, USA). P value of less than $0.05\left({ }^{*} \mathrm{P}<0.05\right.$, $\left.{ }^{* *} \mathrm{P}<0.01,{ }^{* * *} \mathrm{P}<0.001\right)$ was considered as statistically significant difference. 


\section{Cellular Physiology Cell Physiol Biochem 2018;48:1723-1734 \begin{tabular}{ll|l} 
and Biochemistry Published online: August 2, 2018 & $\begin{array}{l}\text { (c) } 2018 \text { The Author(s). Published by S. Karger AG, Basel } \\
\text { www.karger.com/cpb }\end{array}$
\end{tabular} \\ Li et al.: IncRNA GAS5/miR-23a Axis Was Involved in ATG3-Dependent Autophagy and Cell Viability}

\section{Results}

\section{MiR-23a participates in the regulation of ATG3 expression}

Definitely, as an important effector, miRNAs act as negative regulators of gene expression. In order to explore whether miR-23a can target ATG3, we found a potential binding site between miR-23a and the 3'-UTR of ATG3 mRNA using the bioinformatics program (Fig. 1A). We next checked the expressions of miR-23a and ATG3 by transfecting miR-23a mimics or miR-23a inhibitor. The results showed that miR-23a mimics or miR-23a inhibitor could increase or decrease the endogenous miR-23a levels (Fig. 1B and 1C). Meanwhile, ectopic miR-23a suppressed ATG3 expression, and knockdown of endogenous miR-23a induced an increase in ATG3 expression (Fig. 1D and 1E). To understand the effect of miR-23a on ATG3 was specific and the sequence in ATG3 3'-UTR was responsive to miR-23a, we cloned the 3'-UTR sequence of mutant and wild type counterparts into psiCHECK ${ }^{\mathrm{TM}}-2$ dual-luciferase reporter vector. The luciferase reporter assay showed that the wild 3'-UTR of ATG3 exhibited a low level when transfected with miR-23a mimics, whereas the mutant 3'-UTR did not show a response to miR-23a (Fig. 1F). Taken together, these data suggested that ATG3 was a specific target of miR-23a.

\section{MiR-23a inhibits autophagy in vitro}

Forwardly, we wondered whether miR-23a could participate in autophagy in vitro. The 293T cells were transfected with miR-23a mimics or inhibitor, and LC3 mRNA and protein levels were checked by qPCR or Western blotting. The results indicated that miR-23a induced a reduction in LC3- II mRNA and protein expression levels (Fig. 2A and 2B). Besides, as a central regulator of autophagy, mTOR signaling pathway is involved in many cellular processes, such as apoptosis, metabolism, inflammation, and also plays a crucial role in autophagy process. MiR23a overexpression resulted in a significant upregulation of mTOR mRNA and protein (Fig. 2C and 2D). Beclin1 is known as a key regulator of autophagy and mediates the autophagic cell death. Enforced expression of miR-23a significantly reduced Beclin1 protein expression (Fig. 2E). Additionally, miR-23a mimics might inhibit the formation of ATG5-ATG12 complex and increase p62 expression (Fig. $2 \mathrm{~F}$ and 2G). From these results, we concluded that endogenous miR-23a regulated the expression levels of autophagy related genes, suggesting that miR-23a might be involved in autophagy process.

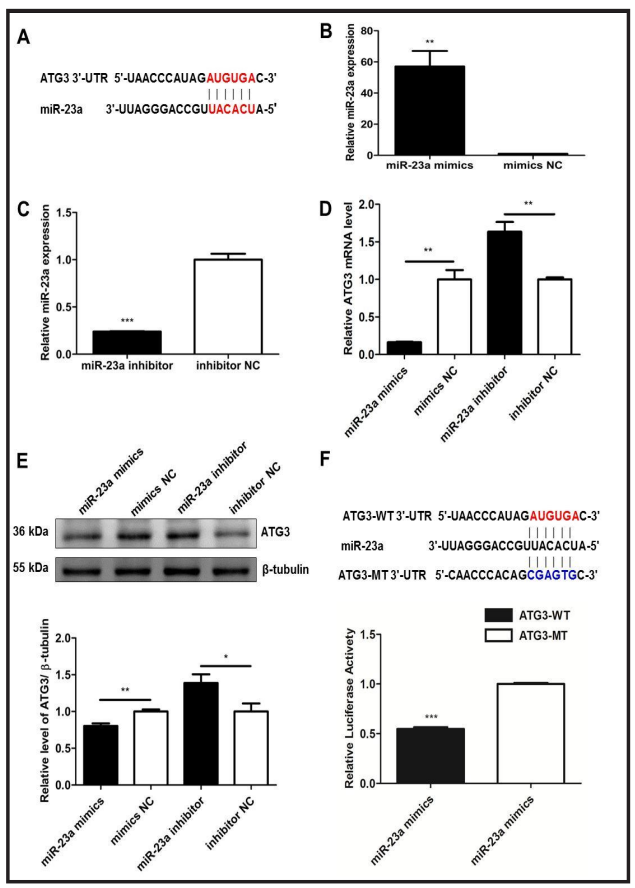

Fig. 1. MiR-23a participates in the regulation of ATG3 expression. Binding site between miR-23a and ATG3 was analyzed using bioinformatics programme. 293T cells were transfected with miR-23a mimics/mimics NC and miR-23a inhibitor/inhibitor NC. RNA and protein were isolated. QPCR and Western blotting were performed as described in the text. (A) Putative miR-23a binding site existed in the 3'-UTR of ATG3. (B and C) Exogenous expressions of miR-23a were detected by transfecting miR23a mimics/mimics NC or miR-23a inhibitor/ inhibitor NC. (D and E) MiR-23a suppressed the mRNA and protein expression of ATG3. (F) Luciferase activity was performed with the indicated cells co-transfected either miR-23a mimics and ATG3-WT or miR-23a mimics and ATG3-MT. Data were shown as mean \pm s.e.m. of three independent experiments $(* \mathrm{P}<0.05$, ** $\mathrm{P}<0.01$, *** $\mathrm{P}<0.001$ ). 

Cell Viability

LncRNA GAS5 is able to directly bind to miR-23a and regulates miR-23a activity

LncRNA has been reported to act as endogenous sponge to interact with miRNA. To understand whether GAS5 could interact with miR$23 \mathrm{a}$ in the autophagic pathway, we noticed that GAS5 contained a binding site of miR-23a using the bioinformatics programme (starBase) (Fig. 3A). Recent studies showed that miRNA negatively regulated IncRNA. We asked whether this was also the case for miR-23a and GAS5. The results indicated that siRNAGAS5 significantly reduced the endogenous GAS5 (the knockdown efficiency was about $85 \%$ ) and increased miR-23a level (Fig. 3B and 3C). In contrast, miR-23a overexpression resulted in decreased GAS5 level (Fig. 3D). To further validate this negative regulation of GAS5
Fig. 2. MiR-23a inhibits autophagy in vitro. 293T cells were transfected with miR-23a mimics or inhibitor. Autophagy related genes of mRNA and protein were analysed by qPCR and Western blotting as described in the text. (A and B) MiR-23a induced a reduction in LC3-II mRNA and protein expression levels. (C and D) MTOR MRNA and protein expression levels were increased after miR-23a mimics was transfected. (E and F) Beclin1 protein levels and ATG5-ATG12 complex formation were decreased, whereas (G) p62 protein level was increased after transfected with miR-
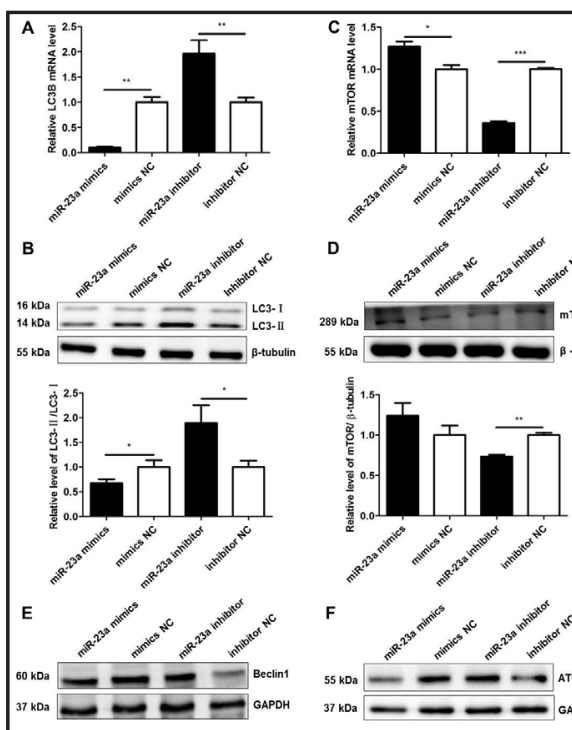

D

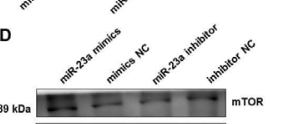

23a mimics. Data were shown as mean \pm s.e.m. of three independent experiments $\left({ }^{*} \mathrm{P}<0.05\right.$, ${ }^{* *} \mathrm{P}<0.01$, $\left.{ }^{* * *} \mathrm{P}<0.001\right)$.
Fig. 3. LncRNA GAS5 is able to directly bind to miR-23a. The binding site between GAS5 and miR-23a was analyzed using the bioinformatics programme. 293T cells were transfected with miR-23a mimics/mimics NC, miR-23a inhibitor/inhibitor NC or pcDNA 3.1-GAS5/pcDNA 3.1, si-GAS5/si-NC. RNA was isolated and evaluated by qPCR as described in the text. (A) MiR-23a-binding site in the GAS5 wild-type form (GAS5-WT) and the mutated form (GAS5-MT) were shown in the upper panel. (B) GAS5 expression was decreased after treated with siRNA-GAS5. The knockdown efficiency was about 85\%. (C) MiR-23a expression was up-regulated following knockdown of GAS5. (D) The binding site in miR-23a was important for miR23a to suppress GAS5. (E and F) The expression of Pri-miR-23a, Pre-miR-23a and mature miR-23a was detected after GAS5 or si-GAS5 was treated. (G) Dual-luciferase reporting vector containing GAS5-WT or GAS5-MT was co-transfected with miR23a mimics or mimics NC in $293 \mathrm{~T}$ cells. The luciferase activity was performed as described in the text. Data were shown as mean \pm s.e.m. of three independent experiments $(* \mathrm{P}<0.05$, ** $\mathrm{P}<0.01, * * * \mathrm{P}<0.001$ ).

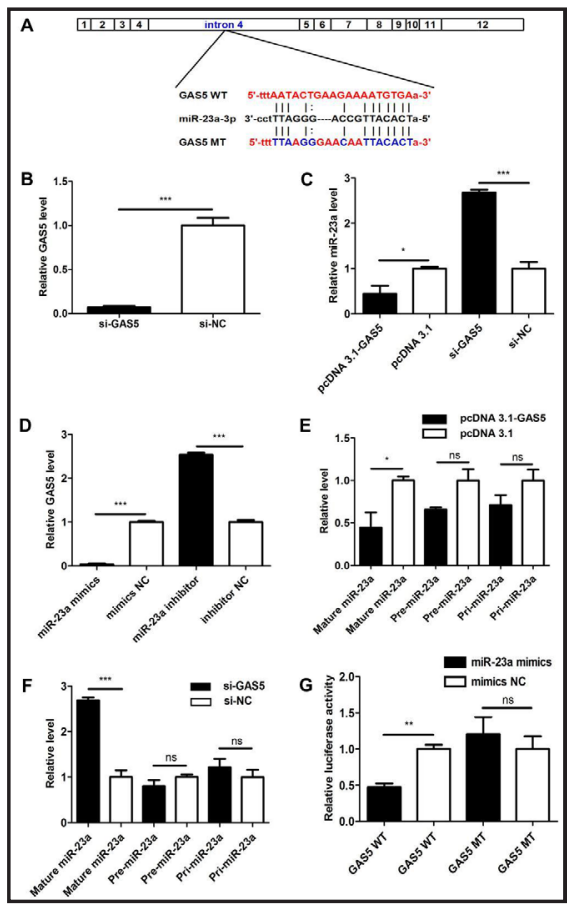


Li et al.: IncRNA GAS5/miR-23a Axis Was Involved in ATG3-Dependent Autophagy and Cell Viability

by miR-23a through a posttranscriptional mechanism, we determined the effect of GAS5 on levels of primiR-23a, pre-miR-23a and mature miR-23a. Ectopic expression of GAS5 induced a significant decrease in mature miR-23a. However, GAS5 had no effect on primiR-23a, pre-miR-23a. On the other hand, knockdown of GAS5 caused the upregulation of mature miR23a but not of pri-miR-23a and pre-miR-23a, indicating that the negative regulation might be through a posttranscriptional mechanism (Fig. 3E and 3F). To determine whether this suppression is through the potential interaction at the putative miR-23a binding site, we produced a luciferase construct of GAS5 (GAS5-WT) and a mutated (GAS5-MT). Luciferase assay revealed that miR23a could suppress the luciferase activity of GAS5, while directed mutagenesis of the predicted miR-23a binding abolished this effect (Fig. 3G). These results demonstrated that GAS5 might interact with miR23a by this putative binding site, suggesting that GAS5 was a function sponger for miR-23a.

\section{Knockdown of GAS5} suppresses the autophagy related genes through miR$23 a$

To further test the functional role of GAS5 in autophagy program, we examined whether the inhibition of GAS5 was capable of affecting autophagy related genes. The results demonstrated that knockdown of GAS5 effectively suppressed the expression of LC3- II mRNA and protein (Fig. 4A and 4B). Subsequently, we
Fig. 4. GAS5 takes part in the regulation of autophagy through miR23a. 293T cells were transfected with siGAS5/si-NC. Autophagy related genes of mRNA and protein were Western blotting as described in the text. (A and B) Knockdown of GAS5 inhibited the LC3II mRNA and protein expression. (C and D) Knowdown of GAS5 resulted in a decrease in ATG3 expression. (E and F) Beclin1 levels and ATG5-ATG12 complex formation were inhibited. ( $G$ and H) MTOR and p62 expression were up-regulated after GAS5 expression was
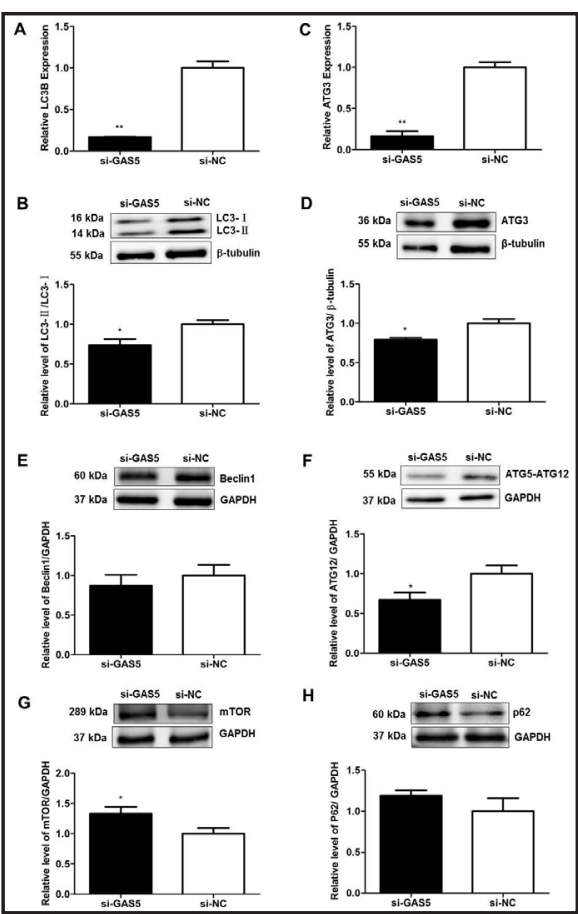

suppressed. Data were shown as mean \pm s.e.m. of three independent experiments $\left({ }^{*} \mathrm{P}<0.05,{ }^{* *} \mathrm{P}<0.01\right)$.

Fig. 5. LncRNA GAS5 and miR23a influence cell proliferation and cell apoptosis. 293T cells were transiently transfected with miR-23a mimics/ mimics NC, miR-23a inhibitor/inhibitor NC or si-GAS5/siNC following LPS stimulation. (A) MiR23a overexpression inhibited 293T cell proliferation and miR-23a inhibitor promoted cell growth.

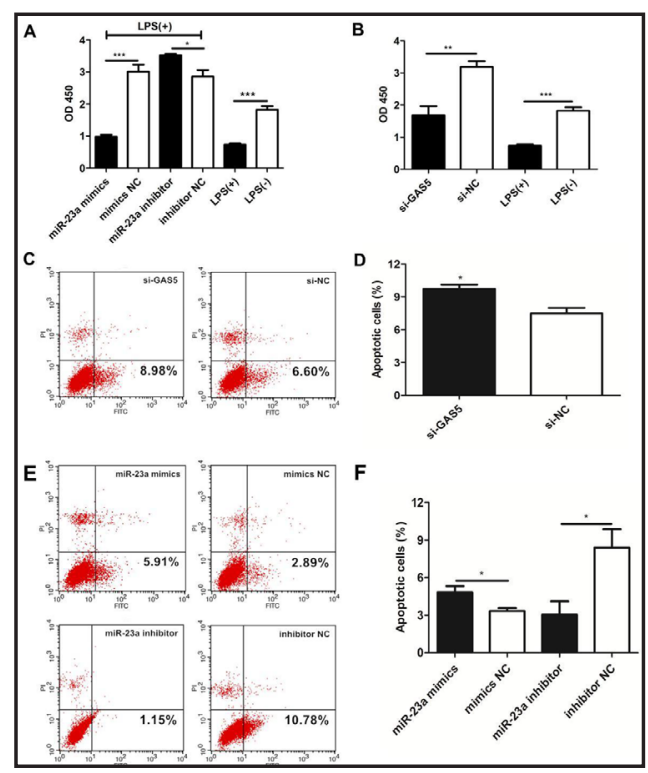

Knockdown of GAS5

also inhibited 293T cell proliferation. (C and D) Down-regulation GAS5 increased cell apoptosis rate. (E and F) MiR-23a mimics increased apoptosis rate. By contrast, the apoptosis rate was suppressed after treated with miR-23a inhibitor. Data were shown as mean \pm s.e.m. of three independent experiments $\left({ }^{*} \mathrm{P}<0.05\right.$, ${ }^{* *} \mathrm{P}<0.01$, $\left.{ }^{* * *} \mathrm{P}<0.001\right)$. analysed by qPCR and 


\section{Cellular Physiology Cell Physiol Biochem 2018;48:1723-1734 and Biochemistry \begin{tabular}{c|c} 
DOI: 10.1159/000492300 \\
Published online: August 2, 2018
\end{tabular} \begin{tabular}{l}
$\begin{array}{l}\text { O 2018 The Author(s). Published by S. Karger AG, Basel } \\
\text { www.karger.com/cpb }\end{array}$ \\
\cline { 2 - 2 }
\end{tabular} \\ Li et al.: IncRNA GAS5/miR-23a Axis Was Involved in ATG3-Dependent Autophagy and Cell Viability}

Fig. 6. Conceptual schematic of GAS5 and miR-23a mechanism of action. ATG3 promotes the lipidation of LC3-I to LC3-IIwith phosphatidyl ethanolamine (PE) during the extension of autophagy membrane. MiR-23a can occupy 3 -UTR of ATG3 binding site to negatively regulate its expression, and block the expression of GAS5. Besides, the mTOR signaling activation is suppressed and Beclin1 expression is inhibited. From the above, all of these results suggest that GAS5/miR-23a/ATG3 axis is involved in autophagy and cell viability.

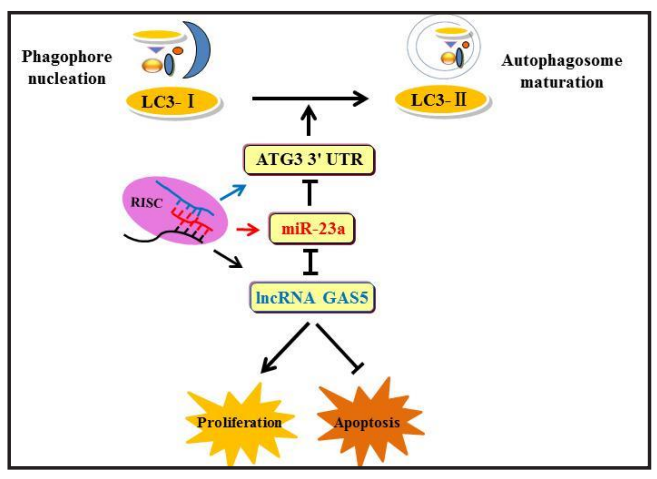

further confirmed whether GAS5 could regulate ATG3, a target mRNA of miR-23a. The data showed that knockdown of GAS5 resulted in a decreased ATG3 mRNA and protein levels (Fig. 4C and 4D). Also, Beclin1 expression and ATG5-ATG12 complex formation were suppressed (Fig. 4E and 4F), and up-regulated expression of mTOR and p62 was observed following si-GAS5 treatment (Fig. 4G and $4 \mathrm{H}$ ). Taken together, these results suggested that GAS5 might target miR-23a/ATG3 axis in the autophagic cascaders and regulate the expression of autophagy key factors.

\section{LncRNA GAS5 and miR-23a participate in cell viability}

It is reported that LPS is involved in cell proliferation and apoptosis $[14,36]$. To further explore the functional role of GAS5 and miR-23a in 293T cells, we tested whether GAS5 and miR-23a were capable of affecting the cell viability and apoptosis. 293T cells were transiently transfected with miR-23a mimics/mimics NC, miR-23a inhibitor/inhibitor NC or siRNA-GAS5/siRNA-NC following LPS stimulation. Cell viability assays indicated that miR23a overexpression inhibited 293T cells proliferation. Contrarily, administration of miR23a inhibitor promoted cells growth (Fig. 5A). We further demonstrated that knockdown of GAS5 also inhibited 293T cells proliferation (Fig. 5B). The results suggested that GAS5 and miR-23a could affect the activity of 293T cells. We next performed apoptosis assays by using flow cytometry with Annexin V-FITC/PI after LPS treated. We observed that downregulation GAS5 increased cell apoptosis rate (Fig. 5C and 5D). The results subsequently showed that miR-23a mimics increased apoptosis rate. On the contrary, the apoptosis rate was suppressed after treated with miR-23a inhibitor (Fig. 5E and 5F). Taken together, these findings suggested that GAS5 and miR-23a were involved in maintaining cell survival.

\section{Discussion}

Autophagy is a cellular self-cannibanlization process and emerges as an important mediator of pathological and pathological responses $[1,2,15,37]$. It serves to preserve the balance between organelle biogenesis, protein synthesis and their clearance [38]. To date, more than 30 ATG proteins and ubiquitin protein system involve in autophagy process [2]. However, emerging evidences have revealed that ncRNAs contribute to autophagy and the regulation mechanism still need to be elucidated [39]. MiR-23a is reported to belong to miR-23a 24-2 27a gene cluster, and is conservative in different cell lines and functions in cell differentiation, tumor growth and metastasis [18-20,40]. The present results showed that miR-23a could target ATG3 which functioned as a E2-like enzyme to participate in conjugating activated LC3- I to phosphatidyl ethanolamine (PE) for lipidation (LC3- II) in the process of extension of autophagy membrane. The luciferase assay and western blotting assay further indicated that miR-23a negatively regulated ATG3 expression resulting in the reduction of LC3- II and Beclin1. Precious study has demonstrated that ATG12 is a target gene of miR-23a. ATG12 usually binds to ATG5 to form ATG5-ATG12 complex contributing 


\section{Cellular Physiology Cell Physiol Biochem 2018;48:1723-1734 \\ \begin{tabular}{l|l} 
and Biochemistry Published online: August 2, 2018 & $\begin{array}{l}\text { C } 2018 \text { The Author(s). Published by S. Karger AG, Basel } \\
\text { www.karger.com/cpb }\end{array}$
\end{tabular} \\ Li et al.: IncRNA GAS5/miR-23a Axis Was Involved in ATG3-Dependent Autophagy and}

Cell Viability

to autophagosome formation [41]. Our results confirmed that overexpression of miR-23a could suppress ATG5-ATG12 complex formation [21]. Additionally, an increased expression of p62 was observed following miR-23a mimics treatment, and the inhibitor abolished the effect. On basis of these findings, the results indicated that miR-23a might represent a novel mechanism to involve in autophagy by targeting to ATG3.

As new regulator, lncRNAs have been affirmed to have considerable functional roles in development, differentiation and metabolism [25]. Increasing evidences have demonstrated lncRNAs are involved in the regulation of autophagic process. For instance, down-regulation of IncRNA MALAT1 attenuated neuronal cell death through suppressing Beclin1-dependent autophagy by regulating miR-30a expression [7]. LncRNA MEG3 suppression could inhibit apoptosis and proliferation through activation of autophagy in T24 cell [42]. GAS5 is originally isolated from the NIH3T3 cells using subtractive hybridization, which exerts its role in anticarcinogenesis, apoptosis and cell growth [43, 44]. Evidences demonstrated that mTOR inhibition can increase GAS5 expression $[33,45]$, and it might be a potential regulator of autophagy [35]. However, the precise regulatory mechanism of GAS5 in autophagy modulation remains largely unclear. Our data investigated that mTOR expression of mRNA and protein was significantly increased with losing the function of GAS5. Moreover, downregulation of GAS5 decreased the expression of LC3, ATG3 and Beclin1. It also suppressed the ATG5-ATG12 complex formation and produced an increased expression of p62. The results seemed that GAS5 expression accounted for the transformation of autophagy levels by mTOR.

It has been demonstrated that IncRNA may act as endogenous sponge RNA to bind miRNA to regulate the expression of miRNA target gene. LncRNA APF could affect the activity of miR-188-3p by targeting ATG7 in myocardial infarction [25]. TUG1 functioned as an oncogenic lncRNA that promoted tumor progression sponging and competing for miR382 binding to the miRNA target EZH2 [46]. LncRNA FLJ11812 could act as a competing endogenous RNA to regulate autophagy in vascular endothelial cells [26]. Recent data showed that IncRNA GAS5 could negatively regulate miR-21 [47]. In this study, the results showed that a significant negative relationship existed between GAS5 and miR-23a. We further found that GAS5 only had an impact on mature miR-23a, but no role in the transcription of primiR-23a and pre-miR-23a, indicating that GAS5 might regulate expression of miR-23a at the post-transcriptional level. According to the prediction of a database (starbase.sysu.edu. cn), GAS5 includes a binding site to miR-23a. The luciferase assay demonstrated that miR23a reduced GAS5 expression through the putative miR-23a binding site in GAS5. Our study further discovered that down-regulation of GAS5 was sufficient to inhibit ATG3 expression through increasing miR-23a expression. The finding indicated that GAS5 might be accessible to target miR-23a and the interaction of autophagy modulation between GAS5 and miR-23a was effective.

As a cellular self-protection mechanism, autophagy can protect cells to prevent metabolic stress and oxidative damage, which benefits to maintain cell survival, cellular homeostasis and the recycling balance of cell products. Many articles implicated that lncRNA could influence the cell viability by activating autophagy. BRAF-activated lncRNA (BANCR) contributed to increases PTC cell proliferation by activating autophagy [28]. Exogenous overexpression of PCGEM1 acted as sponge lncRNA for miR-770 to inhibit apoptosis, induce autophagy and stimulate proliferation in human synoviocytes [48]. Recent report showed that SNHG6 could epigenetically silence p27 and could competitively sponge miR-101-3p thereby regulating zinc finger E-box-binding homeobox 1 (ZEB1) [49]. In this study, miR-23a decreased the cell activity, accompanying with the increment of cell apoptosis rate following LPS stimulation. Additionally, cell proliferation reduced and the apoptosis cells numbers increased by transfecting siRNA-GAS5. This study showed that both miR-23a and GAS5 were able to modulate autophagy, and we inferred that the interaction between miR-23a and GAS5 might regulate cell proliferation and apoptosis by autophagy. 


\section{Cellular Physiology Cell Physiol Biochem 2018;48:1723-1734

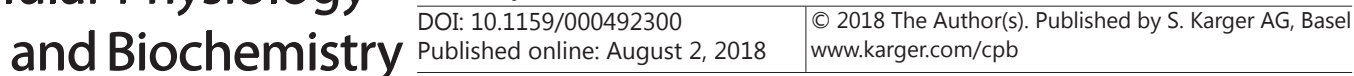 \\ Li et al.: IncRNA GAS5/miR-23a Axis Was Involved in ATG3-Dependent Autophagy and Cell Viability}

\section{Conclusion}

As shown in Fig. 6, our study revealed that down-regulation GAS5 attenuated cell viability and inhibited autophagy through ATG3-dependent autophagy by regulating miR23a expression, suggesting that GAS5 and miR-23a might modulate autophagy and involve in cell viability through their interaction. These results showed that GAS5/miR-23a/ATG3 axis might be a new potential target and biomarker for autophagy regulation and cytoactive study.

\section{Abbreviations}

LncRNAs (long non-coding RNAs); ATG (autophagy related proteins); PI3K (phosphoinositide 3-kinase); PE (phosphatidyl ethanolamine); miRNAs (microRNAs); ceRNAs (competing endogenous RNAs); GAS5 (growth arrest-specific 5); si-GAS5 (GAS5 siRNA); si-NC (negative control siRNA); CCK-8 (Cell Counting Kit-8); LPS (lipopolysaccharide); mTOR (mammalian target of rapamycin); LC3 (Microtuble-associated protein light chain 3); 3'-UTR (3'-untranslated region).

\section{Acknowledgements}

This work was sponsored by National key R \& D program (Grant no. 2017YFD0502301, 2016YFD0501210), Natural Science Foundation of Hubei (Grant no. 2018CFB444), the Fundamental Research Funds for Central Universities of China (Grant no. 2011QC004), and the Open Project Program of the State Key Laboratory of Animal Nutrition of China (Grant no. 2004DA125184F1406).

\section{Disclosure Statement}

The authors declare to have no competing financial interests.

\section{References}

1 Ucar A, Gupta SK, Fiedler J, Erikci E, Kardasinski M, Batkai S, Dangwal S, Kumarswamy R, Bang C, Holzmann A, Remke J, Caprio M, Jentzsch C, Engelhardt S, Geisendorf S, Glas C, Hofmann TG, Nessling M, Richter K, Schiffer M, Carrier L, Napp LC, Bauersachs J, Chowdhury K, Thum T: The miRNA-212/132 family regulates both cardiac hypertrophy and cardiomyocyte autophagy. Nat Commun 2012;3:1078.

-2 Korkmaz G, le Sage C, Tekirdag KA, Agami R, Gozuacik D: miR-376b controls starvation and mTOR inhibitionrelated autophagy by targeting ATG4C and BECN1. Autophagy 2012;8:165-176.

- Kang JW, Cho HI, Lee SM: Melatonin inhibits mTOR-dependent autophagy during liver ischemia/reperfusion. Cell Physiol Biochem 2014;33:23-36.

4 Tekirdag KA, Korkmaz G, Ozturk DG, Agami R, Gozuacik D: MIR181A regulates starvation-and rapamycininduced autophagy through targeting of ATG5. Autophagy 2013;9:374-385.

5 Radoshevich L, Murrow L, Chen N, Fernandez E, Roy S, Fung C, Debnath J: ATG12 Conjugation to ATG3 Regulates Mitochondrial Homeostasis and Cell Death. Cell 2010;142:590-600.

6 Yamada Y, Suzuki NN, Hanada T, Ichimura Y, Kumeta H, Fujioka Y, Ohsumi Y, Inagaki F: The crystal structure of Atg3, an autophagy-related ubiquitin carrier protein (E2) enzyme that mediates Atg8 lipidation. J Biol Chem 2007;282:8036-8043.

7 Guo D, Ma J, Yan L, Li T, Li Z, Han X, Shui S: Down-Regulation of Lncrna MALAT1 Attenuates Neuronal Cell Death Through Suppressing Beclin1-Dependent Autophagy by Regulating Mir-30a in Cerebral Ischemic Stroke. Cell Physiol Biochem 2017;43:182-194. 


\section{Cellular Physiology Cell Physiol Biochem 2018;48:1723-1734

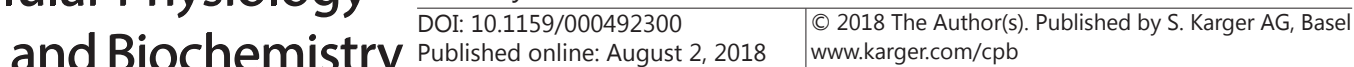 and Biochemistry

Li et al.: IncRNA GAS5/miR-23a Axis Was Involved in ATG3-Dependent Autophagy and Cell Viability

8 Koukourakis MI, Giatromanolaki A, Sivridis E, Pitiakoudis M, Gatter KC, Harris AL: Beclin 1 over- and underexpression in colorectal cancer: distinct patterns relate to prognosis and tumour hypoxia. Br J Cancer 2010;103:1209-1214.

-9 Russell RC, Tian Y, Yuan H, Park HW, Chang YY, Kim J, Kim H, Neufeld TP, Dillin A, Guan KL: ULK1 induces autophagy by phosphorylating Beclin-1 and activating VPS34 lipid kinase. Nat Cell Biol 2013;15:741-750.

10 Bjørkøy G, Lamark T, Pankiv S, Øvervatn A, Brech A, Johansen T: Chapter 12 Monitoring Autophagic Degradation of p62/SQSTM1. Methods Enzymol 2009;452:181-197.

11 Jiang P, Mizushima N: LC3- and p62-based biochemical methods for the analysis of autophagy progression in mammalian cells. Methods 2015;75:13-18.

12 Liu K, Hou Y, Liu Y, Zheng J: LncRNA SNHG15 contributes to proliferation, invasion and autophagy in osteosarcoma cells by sponging miR-141. J Biomed Sci 2017;24:46.

13 Lan SH, Wu SY, Zuchini R, Lin XZ, Su IJ, Tsai TF, Lin YJ, Wu CT, Liu HS: Autophagy Suppresses Tumorigenesis of Hepatitis B Virus-Associated Hepatocellular Carcinoma Through Degradation of MicroRNA-224. Hepatology 2014;59:505-517.

14 Zhang K, Song F, Lu X, Chen W, Huang C, Li L, Liang D, Cao S, Dai H: MicroRNA-322 inhibits inflammatory cytokine expression and promotes cell proliferation in LPS-stimulated murine macrophages by targeting NF-kappaB1 (p50). Biosci Rep 2017;37:1-12.

15 Wang P, Zhang J, Zhang L, Zhu Z, Fan J, Chen L, Zhuang L, Luo J, Chen H, Liu L, Chen Z, Meng Z: MicroRNA $23 \mathrm{~b}$ regulates autophagy associated with radioresistance of pancreatic cancer cells. Gastroenterology 2013;145:1133-1143 e12.

16 Zou Z, Wu L, Ding H, Wang Y, Zhang Y, Chen X, Chen X, Zhang CY, Zhang Q, Zen K: MicroRNA-30a sensitizes tumor cells to cis-platinum via suppressing beclin 1-mediated autophagy. J Biol Chem 2012;287:4148-4156.

17 Lv J, Yang L, Guo R, Shi Y, Zhang Z, Ye J: Ox-LDL-Induced MicroRNA-155 Promotes Autophagy in Human Endothelial Cells via Repressing the Rheb/ mTOR Pathway. Cell Physiol Biochem 2017;43:1436-1448.

-18 Huang S, He X, Ding J, Liang L, Zhao Y, Zhang Z, Yao X, Pan Z, Zhang P, Li J, Wan D, Gu J: Upregulation of miR-23a approximately 27 a approximately 24 decreases transforming growth factor-beta-induced tumorsuppressive activities in human hepatocellular carcinoma cells. Int J Cancer 2008;123:972-978.

19 Zhu Y, Wang D, Wang F, Li T, Dong L, Liu H, Ma Y, Jiang F, Yin H, Yan W, Luo M, Tang Z, Zhang G, Wang Q, Zhang J, Zhou J, Yu J: A comprehensive analysis of GATA-1-regulated miRNAs reveals miR-23a to be a positive modulator of erythropoiesis. Nucleic Acids Res 2013;41:4129-4143.

20 Wang N, Zhu M, Tsao SW, Man K, Zhang Z, Feng Y: MiR-23a-mediated inhibition of topoisomerase 1 expression potentiates cell response to etoposide in human hepatocellular carcinoma. Mol Cancer 2013;12:119.

21 Guo W, Wang H, Yang Y, Guo S, Zhang W, Liu Y, Yi X, Ma J, Zhao T, Liu L, Jian Z, Liu L, Wang G, Gao T, Shi Q, Li C: Down-regulated miR-23a Contributes to the Metastasis of Cutaneous Melanoma by Promoting Autophagy. Theranostics 2017;7:2231-2249.

-22 Gu X, Gao Y, Mu DG, Fu EQ: MiR-23a-5p modulates mycobacterial survival and autophagy during mycobacterium tuberculosis infection through TLR2/MyD88/NF-kappaB pathway by targeting TLR2.

Exp Cell Res 2017; 354:71-77.

-23 Zhang JA, Zhou BR, Xu Y, Chen X, Liu J, Gozali M, Wu D, Yin ZQ, Luo D: MiR-23a-depressed autophagy is a participant in PUVA- and UVB-induced premature senescence. Oncotarget 2016;7:37420-37435.

-24 Tay Y, Rinn J, Pandolfi PP: The multilayered complexity of ceRNA crosstalk and competition. Nature 2014;505:344-352.

25 Wang K, Liu CY, Zhou LY, Wang JX, Wang M, Zhao B, Zhao WK, Xu SJ, Fan LH, Zhang XJ, Feng C, Wang CQ, Zhao YF, Li PF: APF lncRNA regulates autophagy and myocardial infarction by targeting miR-188-3p. Nat Commun 2015;6:6779.

26 Ge D, Han L, Huang S, Peng N, Wang P, Jiang Z, Zhao J, Su L, Zhang S, Zhang Y, Kung H, Zhao B, Miao J: Identification of a novel MTOR activator and discovery of a competing endogenous RNA regulating autophagy in vascular endothelial cells. Autophagy 2014;10:957-971.

27 Yang L, Zhang X, Li H, Liu J: The long noncoding RNA HOTAIR activates autophagy by upregulating ATG3 and ATG7 in hepatocellular carcinoma. Mol Biosyst 2016;12:2605-2612.

28 Wang Y, Guo Q Zhao Y, Chen J, Wang S, Hu J, Sun Y: BRAF-activated long non-coding RNA contributes to cell proliferation and activates autophagy in papillary thyroid carcinoma. Oncol Lett 2014;8:1947-1952.

-29 Wu Y, Lyu H, Liu H, Shi X, Song Y, Liu B: Downregulation of the long noncoding RNA GAS5-AS1 contributes to tumor metastasis in non-small cell lung cancer. Sci Rep 2016;6:31093. 
Li et al.: IncRNA GAS5/miR-23a Axis Was Involved in ATG3-Dependent Autophagy and Cell Viability

-30 Zhang H, Guo Q, Wang X, Wang C, Zhao X, Li M: Aberrant expression of hSef and Sprouty4 in endometrial adenocarcinoma. Oncol Lett 2016;11:45-50.

-31 Mayama T, Marr AK, Kino T: Differential Expression of Glucocorticoid Receptor Noncoding RNA Repressor Gas5 in Autoimmune and Inflammatory Diseases. Horm Metab Res 2016;48:550-557.

-32 Liu D, Dai M, Zhang X, Cao W, Liao M: Subgroup J avian leukosis virus infection of chicken dendritic cells induces apoptosis via the aberrant expression of microRNAs. Sci Rep 2016;6:20188.

-33 Yacqub-Usman K, Pickard MR, Williams GT: Reciprocal regulation of GAS5 lncRNA levels and mTOR inhibitor action in prostate cancer cells. Prostate 2015;75:693-705.

-34 Williams GT, Mourtada-Maarabouni M, Farzaneh F: A critical role for non-coding RNA GAS5 in growth arrest and rapamycin inhibition in human T-lymphocytes. Biochem Soc Trans 2011;39:482-486.

-35 Saxton RA, Sabatini DM: mTOR Signaling in Growth, Metabolism, and Disease. Cell 2017;168:960-976.

-36 Fan WJ, Li HP, Zhu HS, Sui SP, Chen PG, Deng Y, Sui TM, Wang YY: NF-kappaB is involved in the LPS-mediated proliferation and apoptosis of MAC-T epithelial cells as part of the subacute ruminal acidosis response in cows. Biotechnol Lett 2016;38:1839-1849.

-37 Mikhaylova O, Stratton Y, Hall D, Kellner E, Ehmer B, Drew AF, Gallo CA, Plas DR, Biesiada J, Meller J, CzyzykKrzeska MF: VHL-regulated MiR-204 suppresses tumor growth through inhibition of LC3B-mediated autophagy in renal clear cell carcinoma. Cancer Cell 2012;21:532-546.

38 Lee J, Giordano S, Zhang J: Autophagy, mitochondria and oxidative stress: cross-talk and redox signalling. Biochem J 2012;441:523-540.

39 Wang N, Yang L, Zhang H, Lu X, Wang J, Cao Y, Chen L, Wang X, Cong L, Li J, Wang N, Liu Z, Wang L: MicroRNA9a-5p Alleviates Ischemia Injury After Focal Cerebral Ischemia of the Rat by Targeting ATG5-Mediated Autophagy. Cell Physiol Biochem 2018;45:78-87.

40 Cheng L, Yang T, Kuang Y, Kong B, Yu S, Shu H, Zhou H, Gu J: MicroRNA-23a promotes neuroblastoma cell metastasis by targeting CDH1. Oncol Lett 2014;7:839-845.

-41 Romanov J, Walczak M, Ibiricu I, Schuchner S, Ogris E, Kraft C, Martens S: Mechanism and functions of membrane binding by the Atg5-Atg12/Atg16 complex during autophagosome formation. Embo J 2012;31:4304-4317.

42 Ying L, Huang Y, Chen H, Wang Y, Xia L, Chen Y, Liu Y, Qiu F: Downregulated MEG3 activates autophagy and increases cell proliferation in bladder cancer. Mol Biosyst 2013;9:407-411.

43 Mourtada-Maarabouni M, Pickard MR, Hedge VL, Farzaneh F, Williams GT: GAS5, a non-protein-coding RNA, controls apoptosis and is downregulated in breast cancer. Oncogene 2009;28:195-208.

44 Sun M, Jin FY, Xia R, Kong R, Li JH, Xu TP, Liu YW, Zhang EB, Liu XH, De W: Decreased expression of long noncoding RNA GAS5 indicates a poor prognosis and promotes cell proliferation in gastric cancer. BMC Cancer 2014;14:319.

45 Pickard MR, Williams GT: Regulation of apoptosis by long non-coding RNA GAS5 in breast cancer cells: implications for chemotherapy. Breast Cancer Res Treat 2014;145:359-370.

-46 Zhao L, Sun H, Kong H, Chen Z, Chen B, Zhou M: The Lncrna-TUG1/EZH2 Axis Promotes Pancreatic Cancer Cell Proliferation, Migration and EMT Phenotype Formation Through Sponging Mir-382. Cell Physiol Biochem 2017;42:2145-2158.

47 Zhang Z, Zhu Z, Watabe K, Zhang X, Bai C, Xu M, Wu F, Mo YY: Negative regulation of lncRNA GAS5 by miR-21. Cell Death Differ 2013;20:1558-1568.

48 Kang Y, Song J, Kim D, Ahn C, Park S, Chun CH, Jin EJ: PCGEM1 stimulates proliferation of osteoarthritic synoviocytes by acting as a sponge for miR-770. J Orthop Res 2016;34:412-418.

49 Yan K, Tian J, Shi W, Xia H, Zhu Y: LncRNA SNHG6 is Associated with Poor Prognosis of Gastric Cancer and Promotes Cell Proliferation and EMT through Epigenetically Silencing p27 and Sponging miR-101-3p. Cell Physiol Biochem 2017;42:999-1012. 